\title{
Exploring Collaboration with Group Pointer Interaction
}

\author{
Florian Vogt ${ }^{1}$, Justin Wong ${ }^{1}$, Barry A. Po ${ }^{2}$, Ritchie Argue ${ }^{2}$, \\ S. Sidney Fels ${ }^{1}$, and Kellogg S. Booth ${ }^{2}$ \\ ${ }^{1}$ Electrical and Computer Engineering \\ University of British Columbia \\ \{fvogt, jktwong, ssfels\}@ece.ubc.ca \\ ${ }^{2}$ Computer Science \\ University of British Columbia \\ \{po, ritchie, ksbooth\}@cs.ubc.ca
}

\begin{abstract}
Enabling group collaboration is important in computer graphics today. We have developed a framework that supports multiple pointing devices to explore the collaborative utility of multiple mice and laser pointer interaction in graphical environments. Because most pointing device comparisons are done in the context of single user performance, very little is known about the affordances of collaborating with multiple pointing devices. We present an experimental comparison of mouse pointer to laser pointer interaction in a problem-solving task involving groups of one, two, and three people. We show that collaborative performance is largely orthogonal to motor performance and that the interaction patterns are dependent on the task and on the group size. This suggests that the collaborative characteristics of a pointing device are just as important as the physical characteristics that are usually given the most attention, such as precision and accuracy.
\end{abstract}

\section{Introduction}

Interaction with input devices has always been an important part of computer graphics. Using input devices to enable collaboration is a special challenge for Single Display Groupware (SDG) applications and complex graphical environments. Such devices need to be suitable for collaboration, scalable to support groups of multiple people, and capable of supporting social communication for cooperative tasks. These properties are often independent of the factors that most often influence the design of input devices, such as form factor, accuracy, and reliability.

Unfortunately, the collaborative characteristics of input devices are often ignored. Input devices are frequently designed to be used in the context of single users, so usability concerns focus on how the devices can improve single user interaction. As computing becomes more collaborative, it is clear that designers must not only pay attention to the physical ergonomics of input devices, but they must also consider the impact devices will have on display interaction, social communication, and collaborative work processes.

We performed a user study that compared the collaborative aspects of multiple mice and multiple laser pointers in a shared task scenario involving groups of one, two, and three people. We observed that groups chose to cooperate differently depending on whether they were using multiple mice or multiple laser pointers. Moreover, we found that each device conferred different kinds of advantages for group collaboration. This suggests that mice and laser pointers can be used to support different aspects of collaboration and can influence interaction patterns with shared displays.

\section{Background and related work}

For years, pointing devices such as mice have been extensively evaluated in the context of pure interactive performance (i.e. reliability, precision, and accuracy) as they might be used by single users. Recent research has paid more attention to the role that input devices play in group situations. Stewart, Bederson, and Druin [7] introduced the SDG model for supporting collaborative work between co-present individuals. Inkpen et al. [2] investigated the social and productivity benefits of supporting collaborative behavior with children using multiple mice. Stanton, Neale and Bayon [6] evaluated multiple mice and tangible interfaces for encouraging shareable, copresent interaction with children in a desktop environment.

Laser pointer interaction has attracted a great deal of attention in recent years. Olsen and Nielsen [5] adapted existing mouse-based interaction techniques for use with laser pointers. Myers et al. [3] evaluated physical performance differences for laser pointer and distance interaction across multiple form factors. 
Cavens et al. [1] compared the influence of lag and visual feedback on motor performance using mice and laser pointers. Oh and Stuerzlinger [4] introduced a new technique for supporting multiple laser pointers with unique identifiers.

\section{Framework for multiple pointers}

We have successfully implemented concurrent support for multiple laser pointers and multiple mice. Our framework uses a client-server architecture to allow the transparent integration of different devices independent of platform. A pair of UDP socket connections is created between client and server. These connections independently handle button presses and cursor motion events. When supporting a wide variety of input devices, this architecture provides considerable flexibility. Each connected device is uniquely identified and the socket that receives button press events keeps track of the state of all buttons for each device and transmits these to higher-level client applications for processing. Likewise, the socket that receives cursor motion events keeps track of changes in coordinate data and transmits these data to higherlevel client applications for processing.

\section{Objectives}

Because our interactive framework for multiple input devices allowed us to directly compare the collaborative value of multiple mice versus multiple laser pointers, we focused our exploration of collaboration using these two types of devices. Our comparison had three goals:

1. To evaluate the collaborative suitability of multiple mice and multiple laser pointers.

2. To compare the scalability of multiple mice and laser pointers to groups of varying sizes.

3. To investigate the support for social communication with multiple mice and multiple laser pointers.

\section{Methods}

We developed a task scenario where groups of different sizes could use either multiple mice or multiple laser pointers to accomplish a task. The task presented to participants required traversal of a 2D maze to form a shortest path between two designated points in the maze. These maze problems were meant to be solvable by groups of different sizes, from one person to three people. Figure 1 shows the two mazes used in our scenario.
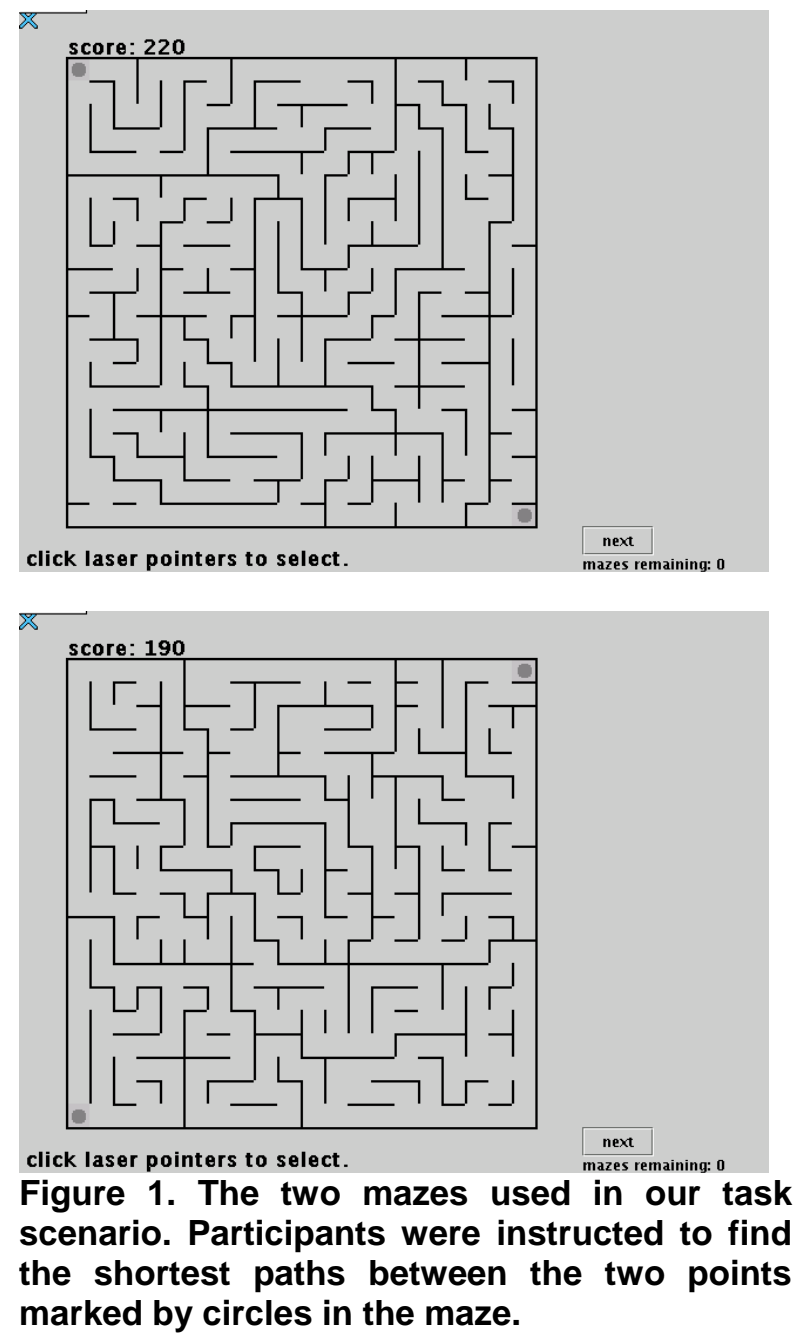

Formally, our experimental design consisted of a single between-groups factor of group size, consisting of three levels (one person, two people, and three people), and a single within-groups factor of interaction style, consisting of two levels (mice and laser pointers). Each combination of group size and interaction style was repeated four times, yielding twelve groups (four groups with one person, four groups with two people, and four groups with three people). The factor of interaction style was fully counterbalanced and groups completed one maze using mice and one maze using laser pointers.

\subsection{Participants}

A total of 24 participants volunteered for our study. Eighteen participants were male and six were female. Ages varied from 20 to 29 years. All participants had normal or corrected-to-normal vision, and were righthanded. The participants were randomly divided into the twelve groups. 


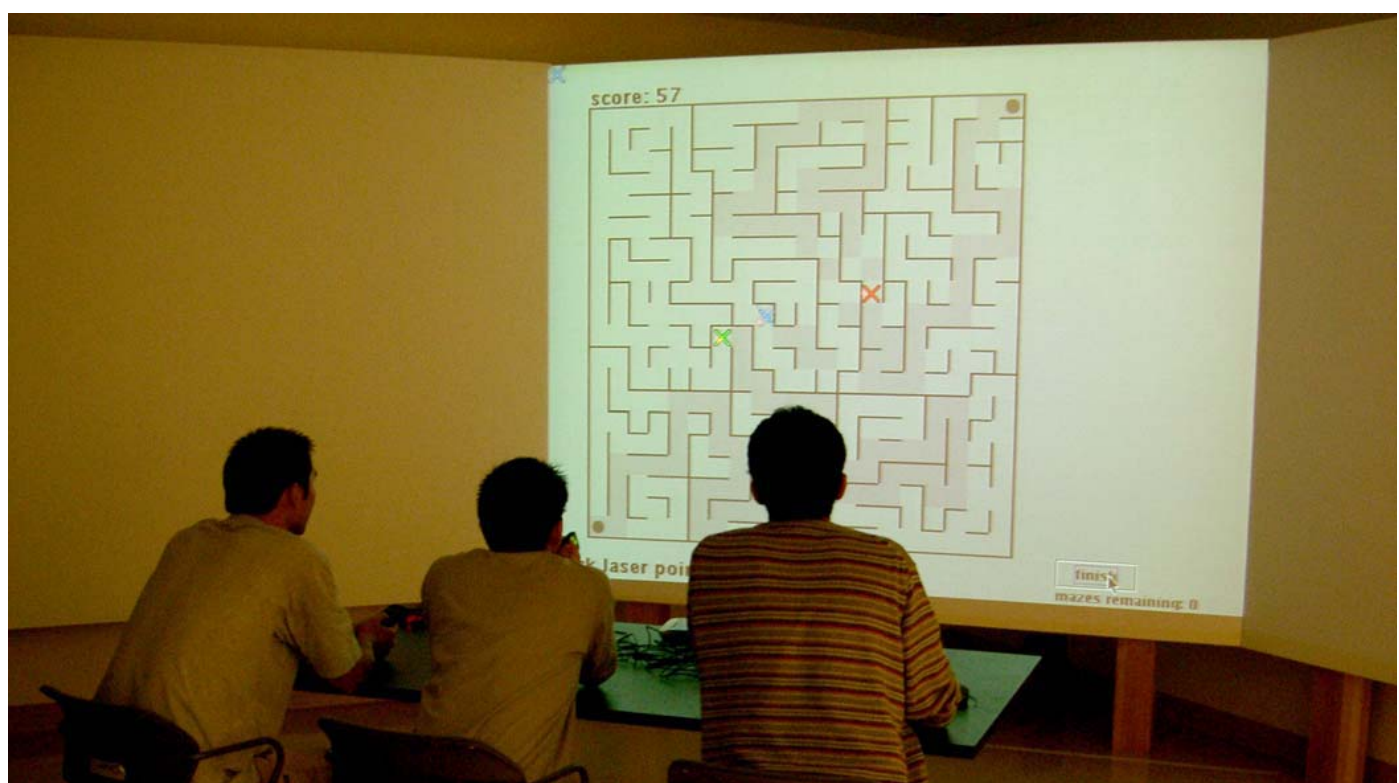

Figure 3. A participant group completing the maze task scenario with laser pointers.

\subsection{Apparatus}

Figure 2 is a photo of the room used during our study. Participants sat beside each other around a rectangular table placed in front of a panoramic, threescreen display. Each screen measured approximately $275 \mathrm{~cm}$ in width and $215 \mathrm{~cm}$ in height. We only used the center screen for our study. The display resolution was scaled to match the resolution of the laser pointer tracker (640x480 pixels) and this resolution was maintained across both mouse and laser pointer interactions.

\subsection{Procedure}

Participant groups were required to complete two mazes, once using mice and once using laser pointers, by finding the shortest paths between a starting point and an ending point in these mazes. The mazes were subdivided into 20x20 pixel squares that could be discretely selected or de-selected. Mazes consisted of 20x20 squares, yielding exactly 400 possible squares to select. The mazes were always presented in the same order so the counterbalancing ensured that half of the groups used laser pointers on the first maze while the other half used mice, and vice versa for the second maze. Both mazes were designed to have similar difficulty. Performance was measured by a score where the highest score was achieved by finding the shortest path using the least number of button presses.

To form a path between two points in a maze, participants were required to select individual squares by pointing at them and pressing a button to indicate selection. Thus, by selecting multiple adjacent squares, groups could connect one point to another. A second button press on a previously selected square would deselect that square. Participants were not allowed to press and hold down device buttons to select multiple squares and form a path by selectively tracing a path from one point to another.

\section{Results}

A variety of quantitative statistical analyses and qualitative observations were used to evaluate the data collected from participants. Interaction logs were used to identify interaction patterns with the multiple mice and multiple laser pointers. These conversation and interaction patterns were used to determine the amount of time that groups spent conversing with one another versus the amount of time they spent using the interaction devices.

\subsection{Group scoring}

Group scores recorded from each maze were standardized to enable a fair comparison between groups. A paired samples $t$-test comparing group score between mouse and laser pointer interaction was statistically significant $[t(11)=2.962 ; p=0.013]$. An evaluation of effect size indicated that mean standardized laser pointer scores were approximately $12 \%$ lower across all group sizes than mouse pointer scores. This is consistent with previous work using laser pointers and mice as single-user interaction devices [3]. 


\subsection{Other observations}

Most participants agreed they found it easier to use the mouse and several suggested this was because of their familiarity with the device. Some participants said they would have preferred to use the laser pointer because they felt the "grounded" nature of the mouse restricted them from moving about, which was something they would have liked to have done. Several other participants mentioned that the direct nature of the laser pointer was easier for collaborating and strategizing, but that the mouse was better for the task of selecting squares, which required finer motor control.

Different group sizes also appeared to affect performance and group behavior. Groups consisting of only one person generally accepted the task to be purely cognitive while groups consisting of three people generally accepted the task to be purely collaborative. Interestingly, neither group size was observed to be faster nor were they necessarily able to complete the task more proficiently. Instead, the real difference appeared to be in how time was spent. Single participants took a more depth-first approach to finding shortest paths: they created small sub-paths, continuing until they came to a final path. Groups of three took a more breadth-first approach to finding shortest paths: they were more likely to identify points along a critical path first as a group before connecting these points together to form a complete path. Groups of two appeared to take an intermediate approach by employing a divide-and-conquer strategy, marking out critical points in the mazes that conveniently divided the maze into two parts that each participant could then solve independently.

\section{Discussion}

The observations made with participant groups suggests that while using multiple mice was superior for the fine motor control aspect of the task scenario, multiple laser pointers were better at encouraging cooperation between participants. This suggests that laser pointers are better suited for collaborative activities involving information sharing and active discussion while multiple mice are better suited for collaborative activities requiring continuous, precise display manipulation or distributed problem solving.

We also observed that multiple mice and laser pointers shared similar scalability characteristics when confined to a Single Display Groupware situation, such as the one used in our task scenario. As group size increased, it became evident that the presence of multiple cursors made it difficult for simultaneous interaction. Several participants from different groups found it difficult to identify the pointer that belonged to them. Moreover, the presence of multiple cursors made it difficult for many participants to focus on the maze task because they were distracted by the dynamic feedback from other pointers. This problem was especially evident for the multiple laser pointers, where dual visual feedback was present. This implies that concurrent manipulation performance with input devices is bounded by the amount of visual feedback on the screen and is entirely independent of the kind of device being used.

\section{Conclusion}

We used a framework for transparently supporting multiple pointing devices to conduct a comparison of multiple mice versus multiple laser pointers in terms of their value as tools for supporting collaboration and social communication. In a maze traversal task involving groups of different sizes, we found that each device promoted different aspects of collaboration and we found evidence suggesting that mice and laser pointers differ in their suitability, scalability, and support for social communication. This suggests that collaborative factors independent of physical device characteristics must be considered when input devices are used in cooperative environments.

\section{References}

[1] Cavens, D., Vogt, F., Fels, S., and Meitner, M. Interacting with the big screen: Pointers to ponder. Ext. Abstracts CHI 2002, 678-679.

[2] Inkpen, K., Ho-Ching, W., Kuederle, O., Scott, S., and Shoemaker, G. This is fun! We are all best friends and we are all playing: Supporting children's synchronous collaboration. CSCL (1999), 252-259.

[3] Myers, B. A., Bhatnagar, R., Nichols, J., Peck, C. H., Kong, D., Miller, R., and Long, A. C. Input Devices: Interacting at a distance: Measuring the performance of laser pointers and other devices. Proc. CHI 2002, 33-40.

[4] Oh, J.-Y., and Stuerzlinger, W. Laser pointer as collaborative pointing devices. Proc. Graphics Interface (2002), 141-149.

[5] Olsen, D., and Nielsen, T. Laser Pointer Interaction. Proc. CHI 2001, 17-22.

[6] Stanton, D., Neale, H., and Bayon, V. Interfaces to support children's co-present collaboration: Multiple mice and tangible technologies. Proc. CSCL (2002), 342-352.

[7].Stewart, J., Bederson, B. B., and Druin, A. Single display groupware: a model for co-present collaboration, Proc. CHI 1999, 286-293. 